Conclusion Recipients of co-located welfare benefits and debt advice experience reduced financial strain and for sub-groups short term mental health is improved. Co-located advice services have the potential to support general practice work but not if co-location is limited to a physical sharing of space. Suggestions are made to facilitate joint working.

\section{PLO2 TOBACCO CONTROL IN ENGLAND: USING MICROSIMULATION MODELLING TO QUANTIFY THE POTENTIAL IMPACT OF A TOBACCO-FREE GENERATION OR A TOTAL BAN}

${ }^{1} \mathrm{C}$ Kypridemos*, ${ }^{2} \mathrm{f}$ Reeve, ${ }^{3} \mathrm{I}$ Buchan, 4,5 A Gilmore, 'S Capewell, ${ }^{1} \mathrm{M}$ O'Flaherty. ${ }^{1}$ Department of Public Health and Policy, University of Liverpool, Liverpool, UK; ${ }^{2}$ WMSSocial Science and Systems in Health, University of Warwick, Coventry, UK; ${ }^{3}$ Farr Institute @ HeRC, University of Manchester, Manchester, UK; ${ }^{4}$ Department for Health, University of Bath, and the UK Centre for Tobacco and Alcohol Studies, Bath, UK; ${ }^{5}$ UK Centre for Tobacco and Alcohol Studies (UKCTAS), Bath, UK

10.1136/jech-2017-SSMAbstracts. 101

Background In 2015, almost one-fifth of English adults continued to smoke. Tobacco control policies in the UK are amongst the strongest in Europe, yet smoking prevalence remains stubbornly high, especially in deprived groups. Novel and radical approaches may be needed to control tobacco effectively. The British Medical Association backs a tobacco ban for those born after 2000, and a population-wide tobacco ban has already been implemented in Bhutan. We use microsimulation modelling to quantify the effectiveness and equity of these two radical tobacco policies.

Methods IMPACT $_{\mathrm{NCD}}$ is a previously validated dynamic stochastic microsimulation developed in $\mathrm{R}$. It simulates the life course and smoking histories of synthetic individuals under alternative scenarios. We used IMPACT $_{\mathrm{NCD}}$ to estimate the potential impact of two proposed changes to tobacco control policy in England - a sales ban restricted to those born in or after 2000 and a total sales ban - on a simulated English population over a 30 year time frame. Extrapolating from Bhutan's implementation, we assumed 50\% reductions in smoking initiation rate, active to ex-smoking ratio, and cigarette consumption. We compared both scenarios with a counterfactual that assumed current declining trends in smoking will continue in the future. We conducted a rigorous sensitivity analysis using second-order Monte Carlo simulation, and we report the median and interquartile range of the output distributions.

Results The model suggested that under the tobacco-free generation caps scenario, smoking prevalence would fall to $12.4 \%$ $(12.1 \%$ to $12.6 \%)$ for men and $7.9 \%(7.7 \%$ to $8.1 \%)$ for women, by 2045 . This could prevent or postpone approximately $3500(-4,200$ to 11,000$)$ cardiovascular disease cases and some $230(-3,100$ to 3,600$)$ lung cancer cases; resulting in approximately $190(-2900$ to 3400$)$ fewer cardiovascular disease deaths, and $220(-2,700$ to 3,200) fewer lung cancer deaths.

Under the total caps scenario, the English smoking prevalence would fall to $7.2 \%(7.0 \%$ to $7.4 \%)$ for men and $4.3 \%$ $(4.1 \%$ to $4.5 \%)$ for women, by 2045 . This could prevent or postpone approximately $90,000(70,000$ to 120,000$)$ cases of cardiovascular disease, some $79,000(55,000$ to 120,000$)$ cases of lung cancer, approximately $14,000(3,000$ to 25,000$)$ fewer cardiovascular disease deaths and some 54,000 (38,000 to 73,000) fewer lung cancer deaths.
Both scenarios could reduce socioeconomic health inequalities in cardiovascular disease and lung cancer morbidity and mortality.

Conclusion Strengthening existing English tobacco control policies through limiting access could substantially improve effectiveness and equity. Further research is now needed to explore the political and legal feasibility issues.

\section{PLO2 PL03 HOSPITAL TREATED DELIBERATE SELF-HARM AND RISK OF SUICIDE AND DEATH FROM OTHER EXTERNAL CAUSES IN THE REPUBLIC OF IRELAND - A NATIONAL REGISTRY COHORT STUDY}

IB O'Farrell*, IJ Perry*, P Corcoran. Epidemiology and Public Health, University College Cork, Cork, Ireland

\subsection{6/jech-2017-SSMAbstracts. 102}

Background Suicide is a major public health problem. The prediction of suicide is difficult, however research has identified that deliberate self-harm (DSH) is one of the strongest predictors of future suicide. To date, the risk of suicide in individuals who DSH is not well established internationally as relatively few countries have accurate data on DSH. This is the first registry based study to examine the risk of mortality on a national cohort of all individuals presenting to hospital due to DSH in Ireland.

Methods A national prospective cohort of 26,168 DSH patients attending the 40 hospital emergency departments in Ireland from 2009 to 2011, were followed up until to the end of 2011 using national death recording systems. Gender specific age adjusted European standardised rates for external cause mortality were calculated. Additionally, Poisson regression was used to generate incidence rate ratios (IRRs). Potential risk factors were investigated using Cox Models.

Results During the study follow-up 437 patients died from external causes. The average 1 year cumulative incidence for suicide, non-suicide external cause mortality and all external causes combined were $0.8 \%, 0.5 \%$ and $1.3 \%$ respectively. The risk of suicide within the first year after DSH was 46 times greater in DSH population compared to the general population. Risk of other non-suicide external cause mortality was also greater in the DSH population compared to the general population (females; IRR $=30$, males; $I R R=20$ ). While the relative risk of death was higher in the female DSH population compared to the female general population, the absolute risk of death was found to be higher in males than females. Older age and male gender were associated with an elevated risk of death. Risk of death from suicide varied depending on method of DSH. Compared with overdose alone attempted hanging had the greatest risk of suicide, particularly in females (females; $\mathrm{HR}=6.8$, males; $\mathrm{HR}=2.6$ ), major self-cutting was also associated with a 2 -fold increased risk. DSH repetition was also found to be a strong predictor of subsequent death.

Conclusion The findings from the world's first national DSH Registry highlight the extremely high risk of death from suicide and other external causes following hospital treated DSH. Older age, male gender, DSH repetition (especially for females) and persons presenting with attempted hanging or major self-cutting are at a particular risk. The findings from this study highlight the need for well-structured, specialist and organised care for this vulnerable group attending emergency departments. 